\title{
ANTIFUNGAL DRUGS RESISTANCE PROFILES OF C. ALBICANS STRAINS ISOLATED FROM THE ORAL CAVITY OF CHILDREN AND ADOLESCENTS
}

\author{
AGNIESZKA KAMIŃSKA ${ }^{1}$, ANNA MALM$^{1}$ and JOLANTA SZYMAŃSKA²*
}

${ }^{1}$ Chair and Department of Pharmaceutical Microbiology with Laboratory for Microbiological Diagnostics, Medical University of Lublin, Lublin, Poland

${ }^{2}$ Department of Integrated Paediatric Dentistry, Medical University of Lublin, Lublin, Poland

\begin{abstract}
Fungi, mainly yeast-like fungi of the Candida genus, and especially the species Candida albicans, occupy an important place among microorganisms that colonize the oral cavity. The aim of this paper was to assess the susceptibility of different strains to some antifungal drugs. The study material consisted of swab samples taken from the buccal mucosa of 376 healthy individuals of both genders, from neonate to18 years. $C$. albicans strains were isolated in 79 persons. To evaluate drug resistance phenotypes of the isolated C. albicans strains Fungitest $^{\mathrm{TM}}$ (Bio-Rad) was used. The study showed that in different age groups the percentage of the tested C. albicans isolates susceptible to all the tested azole drugs varied from $76 \%$ to $95 \%$. Drug-resistant strains were found only in the eldest age group (4.8\%). Phenotypic diversity (drug resistance types) of the studied isolates indicates a wide range of adaptation possibilities enabling colonization of various ecological niches in the oral cavity ecosystem. The presence of $C$. albicans strains intermediate or resistant to azole drugs among the tested isolates may be due to the overuse of that drug group in general therapy and the oral cavity treatment.
\end{abstract}

Keywords: C. albicans, oral cavity, drug resistance, children and adolescents

Fungal diseases of the oral cavity are of opportunistic character. Fungi of the Candida genus, generally considered as physiological components of the oral cavity microbiome, may become, in case of the host's impaired immunity, a causative factor not only of local mycoses but also of multifocal ones. The most frequent factors predisposing children and adolescents to fungal diseases of the oral cavity include: physiological factors - infancy, local disorders - poor local hygiene, high-carbohydrate diet, mechanical lesions in the oral cavity, salivary disorders, the use of orthodontic devices, broad-spectrum antibiotics and steroids, as well as - less frequently - hormonal disorders, neoplasms, immunological defects, xerostomia, and others (1).

The aim of the study was to assess the susceptibility of the $C$. albicans strains isolated from the oral cavity of healthy children and adolescents to the selected antifungal drugs.

\section{EXPERIMENTAL}

The study was approved by the Ethics Committee at the Medical University of Lublin, Poland (decision number: KE 0254/75/2011).
The study material consisted of swab samples taken from the buccal mucosa of 376 healthy individuals of both genders, from neonate to 18 years. The studied subjects were divided into four groups according to the age (neonates, infants, nursery school children - to 3 years) - 102 individuals, 1 (kindergarten children - 4-6 years) - 82 individuals, 2 (elementary school children - 7-14 years) - 91 individuals, 3 (secondary school adolescents - 1518 years) - 101 individuals. The subjects treated with fixed or removable orthodontic devices were excluded from the study. Out of the total number of subjects, $C$. albicans was isolated in 79 persons. The numbers of $C$. albicans strains obtained from each age group were: group $0-17$ isolates, group $1-20$ isolates, group $2-21$ isolates, and group 3-21 isolates.

The culture methods, as well as the results of species identification for the yeast-like fungi isolates, were described in the authors' earlier study (2).

Drug resistance of the isolates was examined using the Fungitest ${ }^{\mathrm{TM}}$ (Bio-Rad) microtest, assessing the susceptibility of the studied $C$. albicans strains to amphotericin B, flucytosine, miconazole, ketoconazole, itraconazole, and fluconazole,

* Corresponding author: e-mail: szymanska.polska@gmail.com 
according to the manufacturer's instruction attached to the kit.

Bio-Rad's FUNGITESTTM Kit was used as a screening method for antifungal susceptibility testing of 79 C. albicans isolates. Six antifungal agents were studied at two different concentrations in modified RPMI 1640 medium. The breakpoints used were 2 and $8 \mathrm{mg} / \mathrm{L}$ for amphotericin B and 2 and 32 $\mathrm{mg} / \mathrm{L}$ for flucytosine. In the case of azole drugs, these values were as follows: 0.5 and $8 \mathrm{mg} / \mathrm{L}$ for miconazole, 0.5 and $4 \mathrm{mg} / \mathrm{L}$ for both ketoconazole and itraconazole and the highest concentrations for fluconazole -8 and $64 \mathrm{mg} / \mathrm{L}$.

Recovering 2 colonies from Sabouraud medium after $24-48 \mathrm{~h}$ incubation at $37^{\circ} \mathrm{C}$, a fungal cell suspension, with turbidity equivalent to 1 McFarland, was prepared in $3 \mathrm{~mL}$ of sterile water. Next, $100 \mu \mathrm{L}$ of the suspension was placed in 1.9 $\mathrm{mL}$ of sterile water and mixed carefully. Using an automatic pipette $20 \mu \mathrm{L}$ of the cell suspension was added into a vial, included in the kit, with the Suspension Medium to obtain $1 \times 10^{3} \mathrm{CFU} / \mathrm{mL}$. Using the pipette, $100 \mu \mathrm{L}$ of the suspension was transferred into each of 8 pairs of microtubes that contained antimycotics and controls. The microtubes were covered with a parafilm and incubated at $37^{\circ} \mathrm{C}$ for $48 \mathrm{~h}$. The results were read visually, by observing the color change into blue or pink.
Susceptibility of a strain to a given drug was shown by the blue color in both corresponding wells. The color change into pink in the first well and blue in the second indicated intermediate susceptibility to amphotericin. The pink color in both wells, corresponding to two different concentrations of the same drug, showed the resistance of a strain to the drug (3-5).

In the next stage of our study, additional tests will be carried out with some antifungal agents according to the CLSI and EUCAST for comparative purposes. We plan to perform E-tests and then MIC (Minimal Inhibitory Concentration) and MFC (Minimal Fungicidal Concentration) of these drugs by the microdilution broth method, using their twofold dilutions in RPMI 1640 broth with MOPS prepared in 96-well polystyrene plates.

\section{RESULTS}

The assessment of the drug resistance of 79 C. albicans isolates showed that the total of 68 $(86.07 \%)$ strains were susceptible to all the examined antimycotics - those strains were described as $\mathrm{S}$ (susceptible). Moreover, all the examined strains were susceptible to two studied antimycotics: amphotericin B and flucytosine. In the individual age groups, the percentage of the $C$. albicans iso-

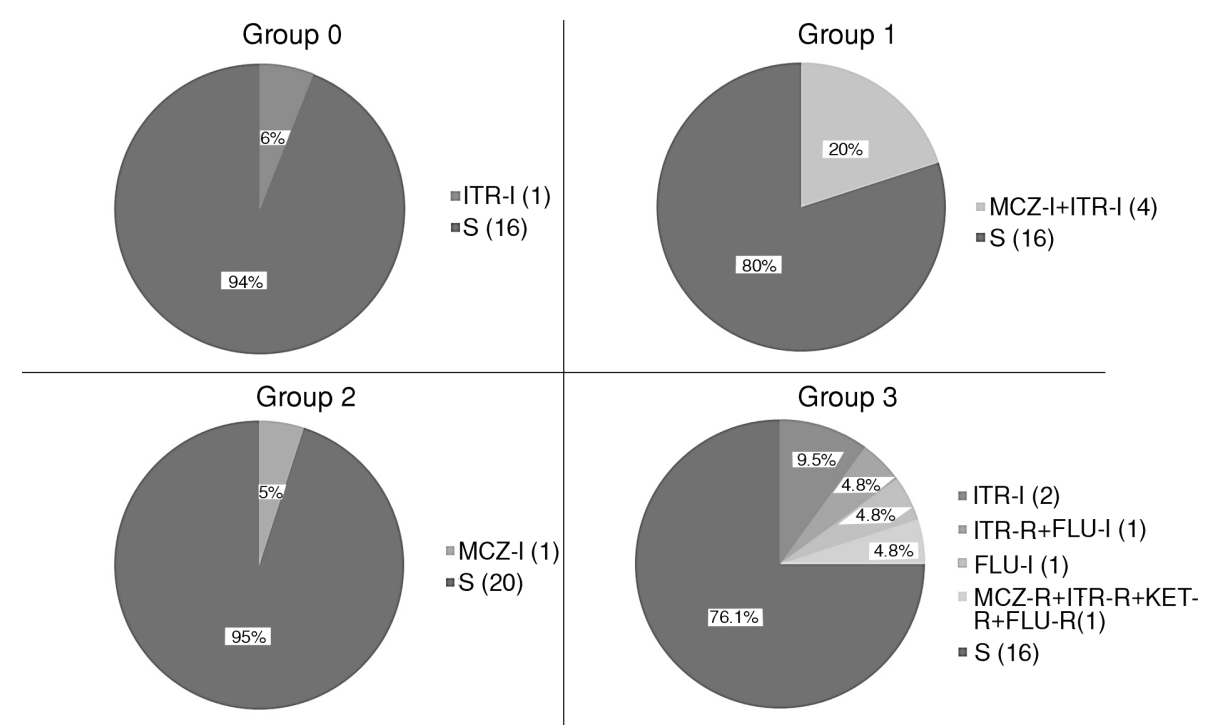

Figure 1. Drug resistance patterns of C. albicans strains isolated from the oral cavity ontocenosis in healthy individuals in individual age groups. Phenotype S (Susceptibility) - a strain susceptibility to all the studied antimycotics; phenotype I (Intermediate) - a strain with decreased susceptibility; phenotype R (Resistant) - resistant; (ITR-I) - decreased susceptibility to itraconazole; (MCZ-I - decreased susceptibility to miconazole; (FLU-I) - decreased susceptibility to fluconazole; (KET-I) - decreased susceptibility to ketonazole; (ITR-R) resistance to itraconazole; (MCZ-R) - resistance to miconazole; (FLU-R) - resistance to fluconazole; (KET-R) - resistance to ketonazole; (ITR-I) - resistance to itraconazole. Group $0-17$ isolates of $C$. albicans, Group $1-20$ isolates of C. albicans, Group $2-21$ isolates of C. albicans, Group 3-21 isolates of C. albicans. 
lates susceptible to all the studied antimycotics varied from $76.1 \%$ to $95 \%$. The isolates with a decreased susceptibility - phenotype I (intermediate) were $5-20 \%$ of all the studied isolates in the individual age groups; the isolates with a decreased susceptibility as well as resistance - phenotype $\mathrm{R}$ (resistant) $-5 \%$ and those showing resistance $-5 \%$. The highest percentage of isolates with a decreased susceptibility was found in group $1-20 \%$ and $3-$ $14 \%$. The presence of drug-resistant strains was detected only in the oldest age group (Figure 1).

\section{DISCUSSION}

Increasing resistance of Candida spp. to the generally used antimycotics such as nystatin, fluconazole, miconazole, itraconazole, and ketonazole, constitutes a serious problem in both local and general treatment of patients with diagnosed mycosis (6-9). The analysis of drug susceptibility of C. albicans and non-Candida albicans strains isolated from the hands of people without any symptoms of disease over a 16-year period in Poland showed increased $C$. albicans and non-Candida albicans strains resistance to commonly used antifungal chemotherapeutics, mainly imidazole. Researchers found a clear rise in susceptibility of $C$. albicans and non-Candida albicans strains to several studied antifungals: 5-fluorocytosine, amphotericin $\mathrm{B}$, miconazole, ketoconazole, itraconazole, and fluconazole (10). The studies by Miskiewicz et al. on the prevalence of oral candidiasis in patients with prosthetic stomatopathy made it possible to evaluate, at the same time, the resistance of C. albicans isolated from dentures to antifungal drugs generally used by dentists. All the cultured C. albicans strains were susceptible to econazole, clotrimazole, miconazole, and nystatin. The resistance of C. albicans to fluconazole was $100 \%$, to ketoconazole $83.3 \%$, and to natamycin $-75 \%$. The cited data point to the co-occurrence of resistance to the most commonly used antifungal drugs, i.e. fluconazole, natamycin, and ketoconazole (11). Similarly, in an Indian study, where a hundred and twenty clinical isolates of $C$. albicans from various clinical specimens were tested for susceptibility against four antifungal agents: fluconazole, itraconazole, amphotericin B, and ketoconazole, a significant increase of drug resistance in clinical isolates of C. albicans was observed (12). Araj et al. conducted a retrospective in vitro study on antifungal drug resistance of, among others, 61 C. albicans strains during three time periods: 2005-2007, 2009-2011, 2012-2014, and found a high resistance (35-79\%) to fluconazole. C. albicans exhibited susceptibility to fluconazole and voriconazole (13). According to other authors, C. albicans isolates obtained from various clinical specimens were susceptible to amphotericin B $(88.8 \%)$ and azole drugs: fluconazole, itraconazole, clotrimazole, and voriconazole (25.5\%) (14).

The present study determined the presence of 6 drug resistance $C$. albicans strains-isolates from the oral cavity of healthy children and adolescents: the greatest number of strains represented the phenotypes corresponding to a decreased susceptibility or resistance to itraconazole This agrees with numerous literature reports stating that the problem of the resistance of yeast-like fungi, including those colonizing the oral cavity, to azole drugs concerns mainly itraconazole (15-19). The resistance of Candida strains to antifungal drugs was found especially in isolates coming from immunodeficient patients (2023). In addition, the results of other studies confirmed a high proportion of $C$. albicans in hospital infections with a very high and high resistance to fluconazole and nystatin, shown by the isolates of C. albicans, as well as Candida krusei and Trichosporon beigelli (24).

It is known that secondary resistance is associated with strains that were originally susceptible and acquired resistance through induction (or selection of natural resistant mutants) (10). Our study showed the presence of drug resistant $C$. albicans strains in the oldest age group (subjects aged 15-18). This may suggest the development of secondary resistance in connection with an earlier local and/or general use of azole antifungals.

As the resistance of $C$. albicans to antifungal drugs commonly used in local and general treatment is increasing, it is necessary to look for simple and adverse affects-free prophylactic and therapeutic means to counteract the increase (11). In the case of oral cavity mycoses, an alternative to conventional antimycotics may be chlorhexidine which, as research confirmed, shows antifungal activity in vivo and in vitro (25).

\section{CONCLUSIONS}

Phenotypic diversity (drug resistance types) of the studied isolates indicates a wide range of adaptation possibilities enabling colonization of various ecological niches in the oral cavity ecosystem. The presence of $C$. albicans strains intermediate or resistant to azole drugs among the tested isolates may be due to the overuse of that drug group in general therapy and the oral cavity treatment. 


\section{Acknowledgments}

The study was financed as part of the statutory activity of the Medical University of Lublin, Poland (grant number: DS 290).

\section{Authors' statement}

The authors declare that they have no competing interests.

\section{REFERENCES}

1. Wójtowicz A., Malm A., Szymańska J.: Mag. Stomatol. 24, 111 (2014).

2. Szymańska J., Wójtowicz A., Malm A.: J. PreClin. Clin. Res. 10, 91 (2016).

3. Krajewska-Kułak E., Niczyporuk W., Łukaszuk C., Krawczuk-Rybak M., Wojtukiewicz M. et al.: Mikol. Lek. 6, 213 (1999).

4. Willinger B., Apfalter P., Hirschl A.M., Makristathis A., Rotter M., Seibold M.: Diagn. Microbiol. Infect. Dis. 38, 11 (2000).

5. Willinger B., Engelmann E., Hofmann H., Metzger S., Apfalter P. et al.: Diagn. Microbiol. Infect. Dis. 44, 253 (2002).

6. Yamashita K., Ohara M., Kojima T., Nishimura R., Ogawa T. et al.: J. Oral Sci. 55, 145 (2013).

7. Nittayananta W., Pangsomboon K., Panichayupakaranant P., Chanowanna N., Chelae S. et al.: J. Oral Pathol. Med. 42, 698 (2013).

8. Mulu A., Kassu A., Anagaw B., Moges B., Gelaw A. et al.: BMC Infect. Dis. 13, 82 (2013).

9. Scott L.J.: Drugs 72, 2141 (2012).

10. Łukaszuk C., Krajewska-Kułak E., Kułak W.: Peer J. 5, e3038 (2017).

11. Miskiewicz A., Szparecki G., Nowak M., Górska R.: Nowa Stomatol. 3, 141 (2013) (in Polish).
12. Zaidi K.U., Mani A., Thawani V., Mehra A.: Mol. Biol. Int. 2016, 4982131 (2016).

13. Araj G.F., Asmar R.G. Avedissaian A.Z.: J. Infect. Dev. Ctries. 9, 997 (2015).

14. Patel L.R., Pethani J.D., Bhatia P., Rathod S.D., Shah P.D.: Natl. J. Med. Res. 2, 439 (2012).

15. Krajewska-Kułak E., Niczyporuk W., Łukaszuk C., Sobaniec H., Wojtukiewicz M. et al.: Mikol. Lek. 7, 27 (2000) (in Polisch).

16. Luque A.G., Biasoli M.S., Tosello M.E., Binolfi A., Lupo S., Magro H.M.: Mycoses 52, 53 (2008).

17. Manfredi M., McCullough M.J., Polonelli L., Conti S., Al-Karaawi Z.M. et al.: Oral Microbiol. Immunol. 21, 177 (2006).

18. Pytko-Polończyk J., Krzyściak P., Macura A.B.: Mikol. Lek. 15, 97 (2008) (in Polish).

19. Resende M., Franco de Sousa L.V.N., Oliveira R.C.B.W., Kogo-Ito C.Y., Lyon J.P.: Mycopathol. 162, 39 (2006).

20. Haddadi P., Zareifar S., Badiee P., Alborzi A., Mokhtari M. et al.: Jundishapur J. Microbiol. 7, e11858 (2014).

21. Shokohi T., Bandalizadeh Z., Hedayati M.T., Mayahi S.: Jundishapur J. Microbiol. 4, S19 (2011).

22. Maheronnaghsh M., Tolouei S., Dehghan P., Chadeganipour M., Yazdi M.: Adv. Biomed. Res. 5, 132 (2016).

23. Badiee P., Badali H., Diba K., Ghadimi Moghadam A., Hosseininasab A. et al.: Curr. Med. Mycol. 2, 24 (2016).

24. Dynowska M., Góralska K., Rosłan M.: Mikol. Lek. 15, 151 (2008).

25. Biernasiuk A., Szymańska J., Kustra A., Korona-Głowniak I., Malm A.: Acta Pol. Pharm. 75, 795 (2018).

Received: 17.02.2019 